\title{
The Geography and Gaze of the Selfie
}

\author{
Matthew Zook, University of Kentucky \\ Ate Poorthuis, Singapore University of Technology and Design
}

Published as:

Zook, M. \& A. Poorthuis (2019). The Geography and Gaze of the Selfie. In: C. Lukinbeal, L. Sharp, E. Sommerland \& A. Escher (Eds.), Media's Mapping Impulse. Stuttgart: Franz Steiner Verlag

\section{Introduction}

Self-portraits have an important place in the history of artistic expression and have been in vogue for centuries. Thus it is not particularly surprising that the selfie - the digital portrait of oneself, most often taken by holding a camera phone at arm's length and subsequently shared through social media - has captured the global public imagination over the last decade. It was declared the Oxford Dictionaries' 2013 Word of the Year (OxfordDictionary, 2013) and has engendered an international research network with over 1500 members (see http://www.selfieresearchers.com/). Given the deep embeddedness of selfies in various social media platforms - they receive more likes and comments than photographs without human faces (Bakhsi et al., 2014) - selfies cannot simply be reduced to images. Instead, we argue that selfies are fundamental to the production and consumption of social media culture, and much more significant than their 4 percent share of imagery might suggest (Manovich, 2014).

In part, the contribution of selfies to the production of social media culture is tied to their geographies. Early selfies were often taken via webcams on desktop computers (i.e., fixed in domestic space) and mostly focus on the photographer's face. Smartphones have untethered the selfie from the home and now the selfie can be as much about the photographer's surroundings as it is about the photographer themselves. What else is within the photo's frame? Who else is included? From where was the selfie sent? This change in character is perhaps best exemplified by the popularity of the selfie-stick. Quite literally, this stick, on which the camera or phone is mounted, enables the user to increase the field of view beyond the photographer's own face. The selfie expands to include the larger moment in space-time that the self is producing around itself. The significance of the geography of the selfie is 
further amplified by the fact that many selfies are explicitly geotagged. That is, users of social media platforms such as Instagram and Twitter specifically choose to tie a specific geographic location to the selfie they are sharing to their friends and followers.

To better understand the selfie phenomenon, we ask 'what is the geography of the selfie?' using a dataset consisting of 8 million geotagged tweets sent from July 2012 to May 2016. Our analysis aims to answer this question using two contrasting and complementary, approaches analogous to the scopic regimes of cartography: projectionism and perspectivalism (Lukinbeal, 2010; Lukinbeal and Sharp, 2016). The 'projectionist' approach (Lukinbeal, 2016; Alpers) provides a view from above. This is the default and now-familiar view offered by much of conventional GIS. In this case, we use quantitative analysis and visualization to understand how the selfie is distributed globally, across space. The second step contrasts this with a perspectivalist approach that offers a situated view representing a gaze from somewhere; contrasting with the god's-eye view of view from nowhere used in the first method. To achieve this, we conduct a qualitative, visual, analysis of the individual selfie images to better understand how selfies connect to specific scenes or geographic contexts and their role in the social production of space.

\section{Social media, the selfie and geography}

While the popular discourse about selfies tends to characterize them as narcissist, attentionseeking and self-promoting (see Figure 1) - perhaps seen as archetypical for the megeneration - academic accounts take a much more nuanced approach. Rather than simply discount selfies as narcissism, scholars build on existing work on social media that identifies a key function of these platforms as the ability to "consume and distribute personal content about the self" (Elison et al, 2011, p. 19). This potential function is particularly useful for celebrities for whom attention is power and "the selfie provides something very powerful, from the most privileged perspective possible... that says, 'Here is a bit of my private life."' (Franco, 2013). Selfies are deployed in a similar manner by other public figures, such as politicians who use them "to experiment with diverse prospects of self-presentation" and enforce or enlarge their political base (Farci and Orefice, 2015, 1). 


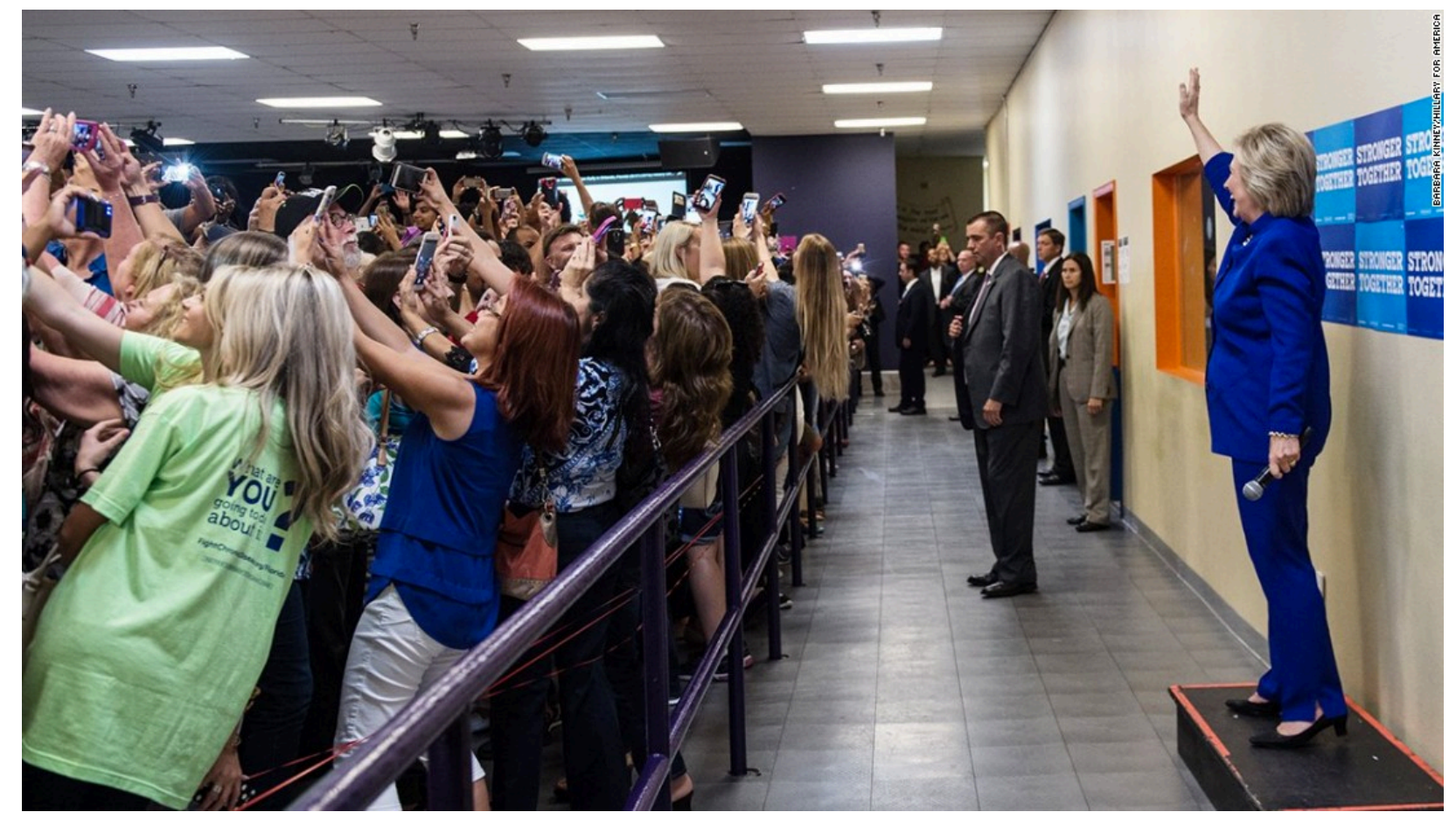

Figure 1: A selfie moment during Clinton's 2016 presidential campaign

For the less-famous, selfies can play a similar role, allowing individuals to share a part of themselves that is regularly hidden (Wargo, 2015) or explore novel practices and new facets of themselves (see Marwick, 2013; Lyu, 2016). Selfies are commonly used within the context of travel and tourism leading Dinhopl and Gretzel (2016) to analyze selfie culture using Urry's (1990) concept of the tourist gaze. They note that, while the traditional tourist gaze was pointed outwards, the technological possibilities and social practices around the selfie have enabled a shift to a more reflective gaze that includes both the tourist destination (i.e. the object) as well as the tourist's relation to such a place (the subject). In fact, they posit that the traditional vistas and views sought by tourists may be subsumed in relative importance as "tourists themselves may become elevated as a touristic product" (Dinhopl and Gretzel, 2016, 135-6). Other scholars echo this evolution of self-representation and have studied the selfie as an art form (Saltz, 2014), a key product of the attention economy (Marwick, 2015) and the ways that selfies are reworking notions of privacy (Busetta and Coladonato, 2015).

Simultaneously, computer scientists and visualization experts have focused on the more quantitative description and visual representation of selfies and those who make them describing demographic characteristics such as age and gender (Souza et al., 2015) or selfie characteristics such as face tilt and mood (Manovich, 2014). An exemplary project following this approach is the Selfiecity (Manovich, 2014) that 'investigates selfies using a mix of 
theoretic, artistic and quantitative methods' with a team of information visualizers, data analysts and web designers. Despite these early efforts, the challenge to understand the "role of the image content in online user behavior" (Bakhsi et al, 2014, 973) is by no means resolved.

The diversity of this scholarship aptly demonstrates that, like any other socio-technical artifact, selfies are not a singular phenomenon; a conclusion also reached by Tifental and Manovich (2015) in their critical reflections on the aforementioned Selfiecity project. In this vein, we suggest that selfies are perhaps best understood as an ongoing conversation - a conversation between the photographer, the people, things and places in physical proximity to the photographer as well as the multitude of people connected through digital means. Perhaps contrary to more conventional conversations, this one explicitly uses visual symbology and culturally determined gestures and practices. Once largely limited to fields such as cultural geography, the use of this type of cultural expression, or texts, are ever more visible in society. As Rose (2016,2 ) argues, "Everyone is reading cultural texts and coming to conclusions about their meaning and sharing their interpretations ... [about] ... the symbolism of specific cultural texts but also the production and circulation of those texts by specific forms of media institutions." This academic formulation is aptly captured by the observation of celebrity James Franco (2013) - the so called selfie king - who voices much the same sentiment about the presences of cultural interpretation in everyday digital culture: "A texting conversation might fall short of communicating how you are feeling, but a selfie might make everything clear in an instant ... I want to know whom I'm dealing with. In our age of social networking, the selfie is the new way to look someone right in the eye and say, "Hello, this is me." If the selfie is 'worth a thousand words', we attempt to unpack what these words might mean in this chapter.

Despite the prevalence of selfies, few scholars have explicitly studied the spatiality of the selfie. This is particularly noteworthy given the attention given to the geography of social media more generally, ranging from relatively trivial examples - such as the distribution of tweets about particular brands of beer (Zook and Poorthuis, 2014) - to more noteworthy issues such as spatial segregation within cities and neighborhood identification and formation (Shelton et al, 2015). Scholars outside of geography - primarily sociology - also analyze the spatiality of social media, for example in the ways that time is flattened in favor of interfaces that "strongly emphasizes physical place and users' locations" (Hochman and Manovich, 
2013) or the ways that its practice is used to "showcase patronage of exclusive and expensive places" (Boy and Uitermark, 2015). More specifically related to imagery, Hjorth and Hendry (2015) outline an idea of "emplaced visuality" that places movement (and thereby space and time) at the center of how people utilize digital media.

Even with these exceptions, there remains little systematic study of the geographies of this key practice and producer of digital culture. This chapter addresses this gap by explicitly focusing on the spatiality of the selfie. We do so by both using quantitative approaches resulting in a view from 'nowhere' akin to traditional topographic maps - as well as a qualitative analysis of the selfies as cultural text, yielding a view from 'somewhere' that can complement and complicate the quantitative mapping.

\section{Methods \& Data}

Social media data differs from more traditional census and survey datasets in a number of key ways. There are specific advantages (e.g. social media generally have much higher spatial and temporal granularity) and important downsides (e.g. social media data does not represent the entire population and its content is unstructured and open to interpretation). Thus, research with social media must take care in the type of questions and the specific analyses conducted. For this reason, much of our previous research treats social media largely as a medium, or a mere proxy for activity or attention associated with particular criteria (e.g., the spatial variation of tweets containing the term grits, (Poorthuis et al, 2016)) rather than grappling with the content and purpose of the message and its connection to space.

Analyzing the actual content of social media messages is a difficult task given the slangheavy shorthand common within much social media, the reliance on images rather than text, as well as technical constraints of the medium itself, e.g., the 140 character limit on tweets. Despite these difficulties it is worth pursuing, as social media data potentially offers insight on types of human behavior - a more vernacular geography - that hitherto have been extremely problematic to pursue. Precisely because social media allows individuals to document the everyday banalities of life, it is possible to study some practices - discourse with friends and family, interacting with the built environment, etc. - at a breadth and scale that was simply not possible otherwise. Of course, in addition to the textual messiness of such data, researchers must also confront the issue that social media is not an unfiltered or true 
representation of an individual's life, as people may curate their representations on social media to achieve multiple aims.

This chapter uses the DOLLY database at the University of Kentucky - a global dataset of geotagged tweets from July 2012 to May 2016 (Poorthuis and Zook, 2016) - to produce a corpus of selfies to analyze. To build this collection, we selected all tweets within this dataset that include the term "selfie" (in 20 different languages ${ }^{1}$ ); a total of 8.1 million tweets. While acknowledging that Twitter is but one form of social media and selfies are distributed via a number of other platforms - Facebook, Instagram, other messaging platforms - this approach has a number of advantages. First, and most importantly, it is an accessible source of selfies with global coverage. While this coverage is undoubtedly uneven, the broad contours of this bias are somewhat known, e.g., Twitter is not a widely used platform in China because of the Great Firewall, and therefore results can be interpreted in this light. Second, it is also possible to draw a random sample of all tweets within the aforementioned dataset to serve as a normalization variable. This allows us to gauge the frequency of selfies relative to all Twitter messages. Third and finally, it provides archived imagery that can be drawn upon for analysis. We are well aware of the short-comings and issues surrounding the use of Twitter data for social science research (cf. Crampton et al., 2013) and interpret our findings only as representative of selfie activity within geotagged tweets. Moreover, given the variety of media in which selfies are set, the fact that many of these media are private or are on platforms that do not allow access via an API (e.g. Facebook), it is simply impossible to create a wholly representative corpus of selfies. The approach used here, however, offers an important case study of the spatial practices of selfie on a major social media platform.

With a corpus of 8.1 million selfie-related tweets ready, we embark on an analysis of the geography of the selfie based on the approaches outlined in the previous section. In the projectionist - or view from above - approach we analyze and visualize the geographic distribution of selfie production to examine what parts of the world are the most prolific

\footnotetext{
${ }^{1}$ Although many languages use the English loanword, we also use the following other translations of 'selfie' in some of the world's more popular languages (based on the 25 most spoken languages (SIL, 2015)): "selfie", “селфи”, “" “" “', “autofoto “, “selfi”, “Selfy”, “ সেলফি “, “Sebić “, “Szelfi “, “Swafoto “, “自分撮り”, “

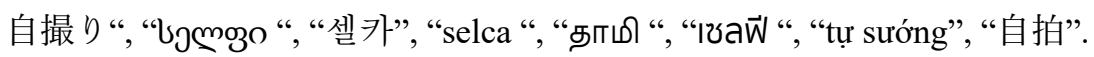


producers of selfies. An specific advantage of drawing upon geotagged tweets is that each tweet is tied to a specific point in space with a set of lat/lon coordinates. This allows us to aggregate and normalize data at different scales and using different areal units. While standard definitions (e.g., countries) are employed as useful containers for interpreting results (see Table 1), we can also use identically-sized units (e.g., the hexagons we use here), which aid in the visual interpretation of the maps in the next section.

The geographic analysis of social media always necessitates a standardization or normalization step, lest we simply end up studying population density. In this study we normalize the number of selfie tweets by the number of overall tweets at that same location to distinguish between relatively higher and lower levels of selfie activity. The exact metric we use here is the odds ratio (Bland and Altman, 2000; Poorthuis et al, 2016) which can be interpreted as follows: locations that score 1 have exactly the number of selfie tweets one would expect given the total number of overall tweets in that location, while places with scores either more than or less than 1 have relatively more or less selfie activity than the norm. To guard against the issue of small sample sizes - a particular problem for sparsely populated areal units - we also use 95 percent confidence intervals to ensure that any concentrations of high selfie activity shown in the map are statistically significant.

In the perspectivalist approach, we conduct a qualitative and visual analysis of the actual selfie images, using Rose's critical visual methodology (2012) to understand how the visual and textual context of selfies (re-)produces digital and material cultures. To do this, we first draw a random sample of 1000 selfie tweets from the corpus and confirm (via an automated script) that each selfie indeed (1) contains an image or photo; (2) is still publicly available on Twitter; and (3) is still publicly available (usually accessed via a URL in the text of the tweet) on a third-party service, such as Instagram, if applicable. Twitter users have the option of either deleting tweets or making them private and the company itself can remove material. While it is often possible to access the image associated with a deleted tweet (as it is stored elsewhere) good ethical research within social media respects the intentions of individuals.

With this random sample of public tweets identified, the script automatically takes a screenshot of the selfie tweet including imagery, associated text and context (replies, likes, etc.). Taking the first 50 tweets from this sample both authors reviewed each screenshot to 
create a coding system that emerged organically from the screenshots. Our intent was to construct categories that could realistically be coded reliably, reflected the variety of intent and representation found within selfies and could give meaningful insight to our research questions. Our categories include the following: number of people, age $(<30,>30$, multiple/unknown), gender (M, F, multiple, unknown), Place (none, banal, significant), Event (none, banal, significant), Posture (purposeful, accidental), tools (mirror, stick, collaging), self-image (fashion, health), meta (commentary, art, funny, solicitation).

We very much recognize that this is not a definitive list of relevant variables and that was not our goal. We also do not believe that our coding is unproblematic. Selfies are complex cultural objects that do multiple and contradictory work in the reproduction of society. The meaning or message intended by the creator is often difficult to discern beyond a fairly superficial level. Our intent therefore was to experiment with what was possible in interpreting these type of images (e.g., judging whether an event was significant also relied on our ability to read the language of the associated text) and subsequently (and more importantly) identify a broad set of meanings and work that selfies were doing in the production of digital culture.

Having established our codebook, we then randomly selected another 215 selfies from the two countries with the most selfies in absolute numbers - the United States and Indonesia (see Table 1. We focused our sample on these two countries rather than taking a global sample in order to better understand specific regional practices - granted a somewhat quixotic goal given the large population bases of both - and also to identify possible differences between places. This echoes Souza et al's (2015) quantitative analysis of selfies, albeit their approach was based on an algorithmic coding of millions of selfies with the goal of explaining inter-country variation via cultural and socio-economic indicators. While we are intrigued by their finding that countries with lower measures of gender equality have fewer women posting selfies (which they interpret as meaning "selfies mobilize the power dynamics of representations and promotes empowerment") we have different aims. Responding in part to Rose's $(2016,4)$ call to attend to the "thoughts, feelings, processes and practices which are then mediated by such technologies" and given the multiple issues with this data - ranging from its biased sampling to the imprecision of our coding - we are cautious in our interpretations, seeing them as suggestive of possible trends and differences 
that warrant more systematic study via qualitative and ethnographic techniques. In the subsequent sections, we present the results of both of these approaches.

\section{The Geography of Selfie}

A cursory look at the straightforward mapping of the raw number of selfie-related tweets in Figure 2, reveals one immediate insight: as expected, the selfie phenomenon has spread far and wide across the globe. Selfies are found pretty much anywhere where Twitter as a platform is used. Selfies are not only sent from global urban centres but from places that range from the American Deep South to Tokyo's metropolis and from around the entire Mediterranean to the South American Pampas. When we normalize the raw count in each hexagon a more nuanced (and somewhat more unexpected picture) emerges (Figure 3). Although in much of the Anglophone popular media selfies are portrayed as a favorite pastime of Western suburban teenagers, we find that selfies represent a much higher proportion of overall Twitter activity in other regions of the world. Central America, Eastern Europe, parts of the Middle East and South and South-East Asia contain a very high number of selfies relative to the total number of tweets.

3

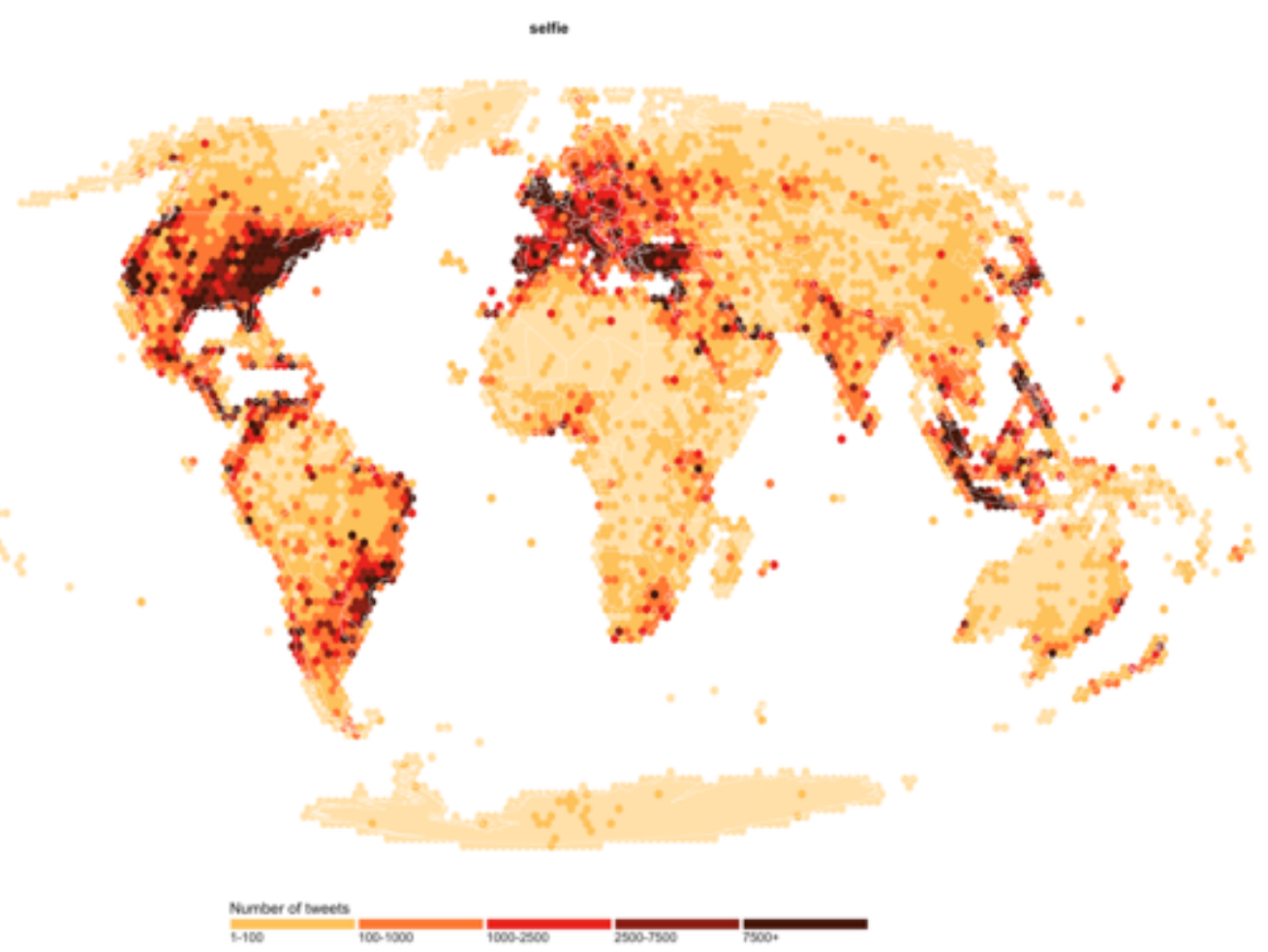

Figure 2: Selfie-related tweets (count) 


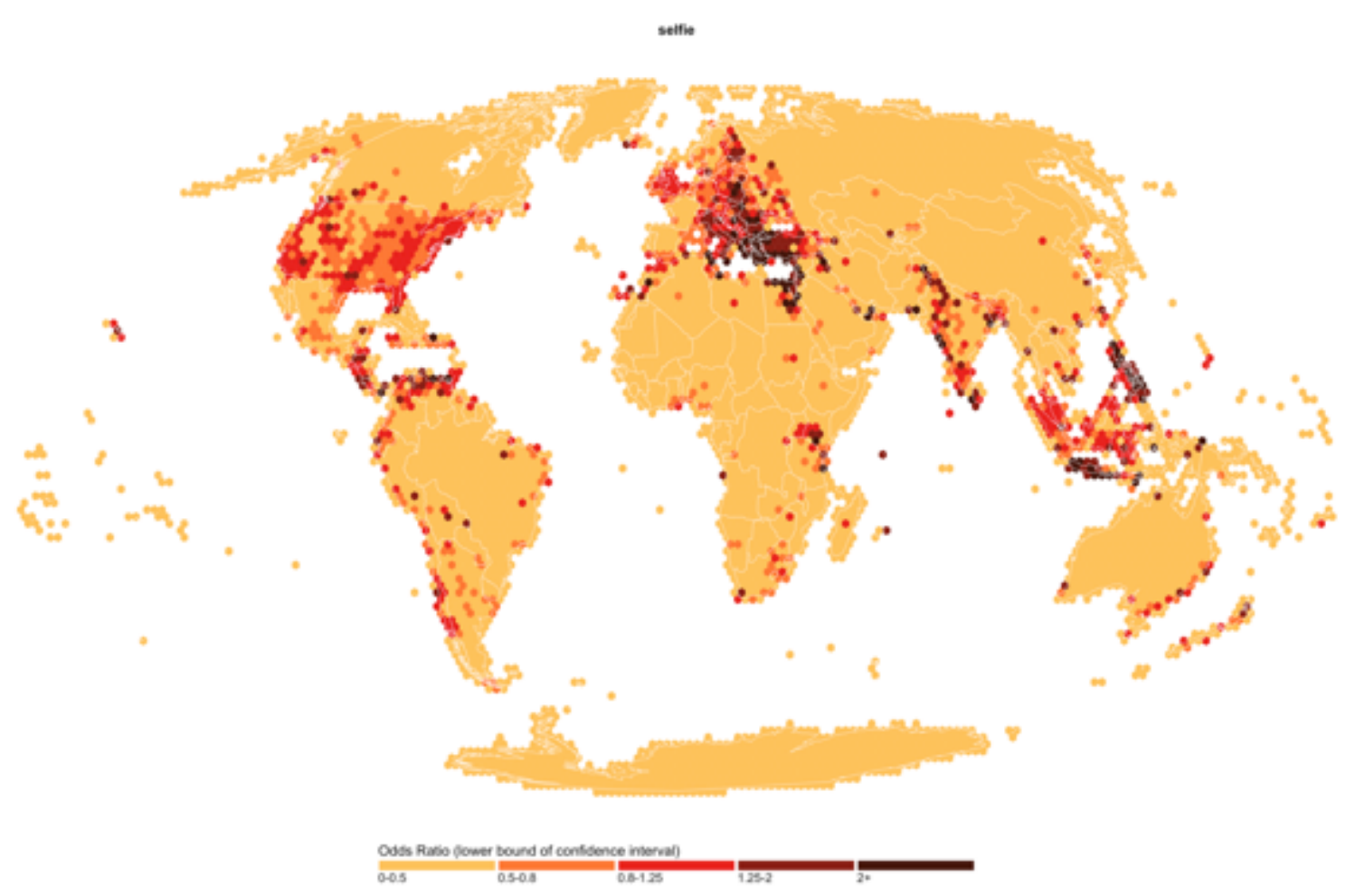

Figure 3: Selfie-related tweets (odds ratio)

Zooming in from the global scale to a more regional scale in South-East Asia (Figure 4), it becomes abundantly clear that selfie culture is not a predominantly urban phenomenon. In all of the region's countries in which Twitter is a popular platform, find prolific selfie production in cities such as Jakarta, Singapore and Kuala Lumpur and the countries' hinterlands. High odds ratios are found throughout the Indonesian islands of Borneo, Sulawesi, Java and Sumatra and the entire countries of Malaysia and the Philippines. 


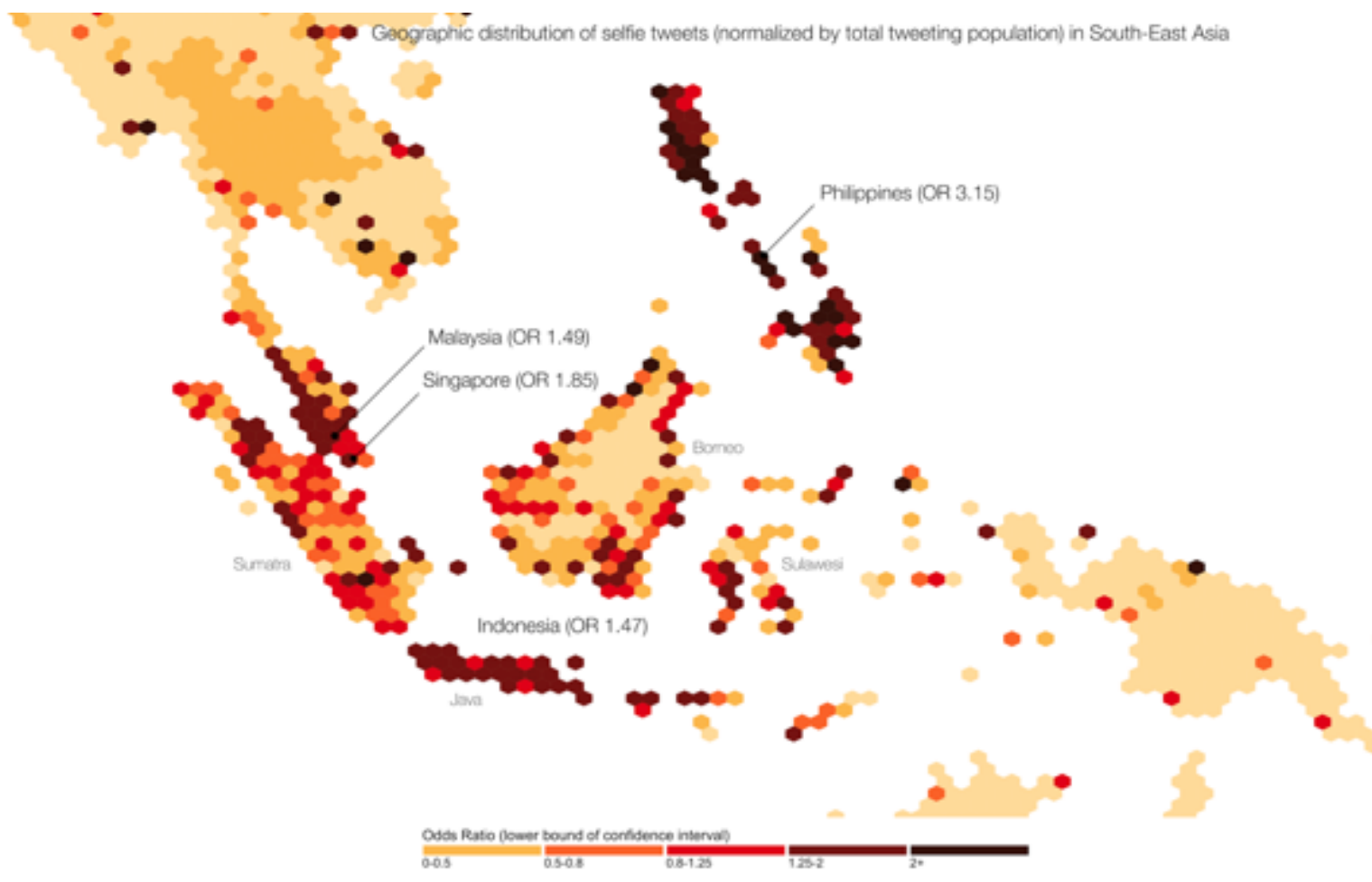

Figure 4: South East Asia

Similar trends can be seen in Table 1 containing both the raw number of tweets as well as the normalized odds ratio per country instead of hexagon. Based on the raw count, the top 15 countries holds a lot of the 'usual suspects', including the US, UK and France. However, the top 15 countries for the odds ratio measure is completely different and now home to many countries that are perhaps often considered to be at the periphery of global social media production and participation.

\begin{tabular}{|l|l|}
\hline \multicolumn{2}{|c|}{ Rank based on \# of selfies } \\
\hline 1 & USA \\
\hline 2 & Indonesia \\
\hline 3 & Turkey \\
\hline 4 & Brazil \\
\hline 5 & United Kingdom \\
\hline 6 & Philippines \\
\hline 7 & Malaysia \\
\hline 8 & Spain \\
\hline
\end{tabular}

\begin{tabular}{|l|l|}
\hline \multicolumn{2}{|l|}{ Rank based on Odds Ratio } \\
\hline 1 & Bangladesh \\
\hline 2 & Philippines \\
\hline 3 & Cambodia \\
\hline 4 & Slovakia \\
\hline 5 & Sri Lanka \\
\hline 6 & India \\
\hline 7 & Vietnam \\
\hline 8 & Mauritius \\
\hline
\end{tabular}




\begin{tabular}{|l|l|}
\hline 9 & Italy \\
\hline 10 & Argentina \\
\hline 11 & Mexico \\
\hline 12 & Japan \\
\hline 13 & Russia \\
\hline 14 & Canada \\
\hline 15 & France \\
\hline
\end{tabular}

\begin{tabular}{|l|l|}
\hline 9 & East Timor \\
\hline 10 & Lebanon \\
\hline 11 & New Zealand \\
\hline 12 & Romania \\
\hline 13 & Australia \\
\hline 14 & Taiwan \\
\hline 15 & Czech Republic \\
\hline
\end{tabular}

Table 1, Summary of country-level rankings

To explore this issue further, and reflecting upon the work by Souza et al. (2015), we thought it useful to undertake a similar, albeit much simpler, country level analysis of indicators that correlate strongly with the calculated odds ratios (See Table 2). We found that measures of gender equality preformed best and countries with high equality ratings (including women's literacy) also had a higher than average production of selfies. These correlations were much stronger than income - a more typical relationship seen with information technology suggesting that selfies are indicative with women's empowerment at this scale. While we think it is unlikely that selfies are directly causing equality, the ability to engage with the practice of selfie seems consistent with larger societal trends particularly as they emerge in digital cultures.

\begin{tabular}{|l|c|}
\hline Variable & Correlation \\
\hline $\begin{array}{l}\text { Gender equality rating (1=low to } \\
6=\text { high) }\end{array}$ & 0.294 \\
\hline Mobile phones per 100 people & 0.237 \\
\hline Literacy rate, adult women & 0.227 \\
\hline $\begin{array}{l}\text { Scientific journal articles } \\
\text { published per capita }\end{array}$ & 0.146 \\
\hline GNI per cap & 0.062 \\
\hline Mobile, percent women poor & 0.027 \\
\hline Mobile, percent women & 0.005 \\
\hline $\begin{array}{l}\text { Percentage of women who believe } \\
\text { a husband is justified in beating his }\end{array}$ & -0.227 \\
\hline
\end{tabular}




$$
\text { wife }
$$

Table 2, Country level correlations between Odds Ratio for Selfies and World Bank

$$
\text { indicators }
$$

\section{The Gaze of the Selfie}

Shifting our analysis from the quantitative view-from-above to a more qualititative, subjective view-from-somewhere allows us to better understand how selfies are involved in the production of those spaces. To ground this approach relatively to previous work, we begin with a closer look at the aforementioned Selfiecity project (Manovich, 2014). The dataset underlying this project was constructed by taking a set of randomly selected photos from Instagram and having multiple Amazon Mechanical Turk workers code whether the image was a selfie or not. The result can be seen in Figure 5. The selfies in the selfiecity project are surprisingly uniform. There are variations from city to city (in inferred gender, age, head tilt and 'smile score') but overall the result is a uniform grid of individual faces. It is this representation that is so popular as the archetypical version of the selfie: the "me, me, me' devoid of any context, relations to other people or connections to place. Our reading and analysis of selfies, however, generates radically different results. 


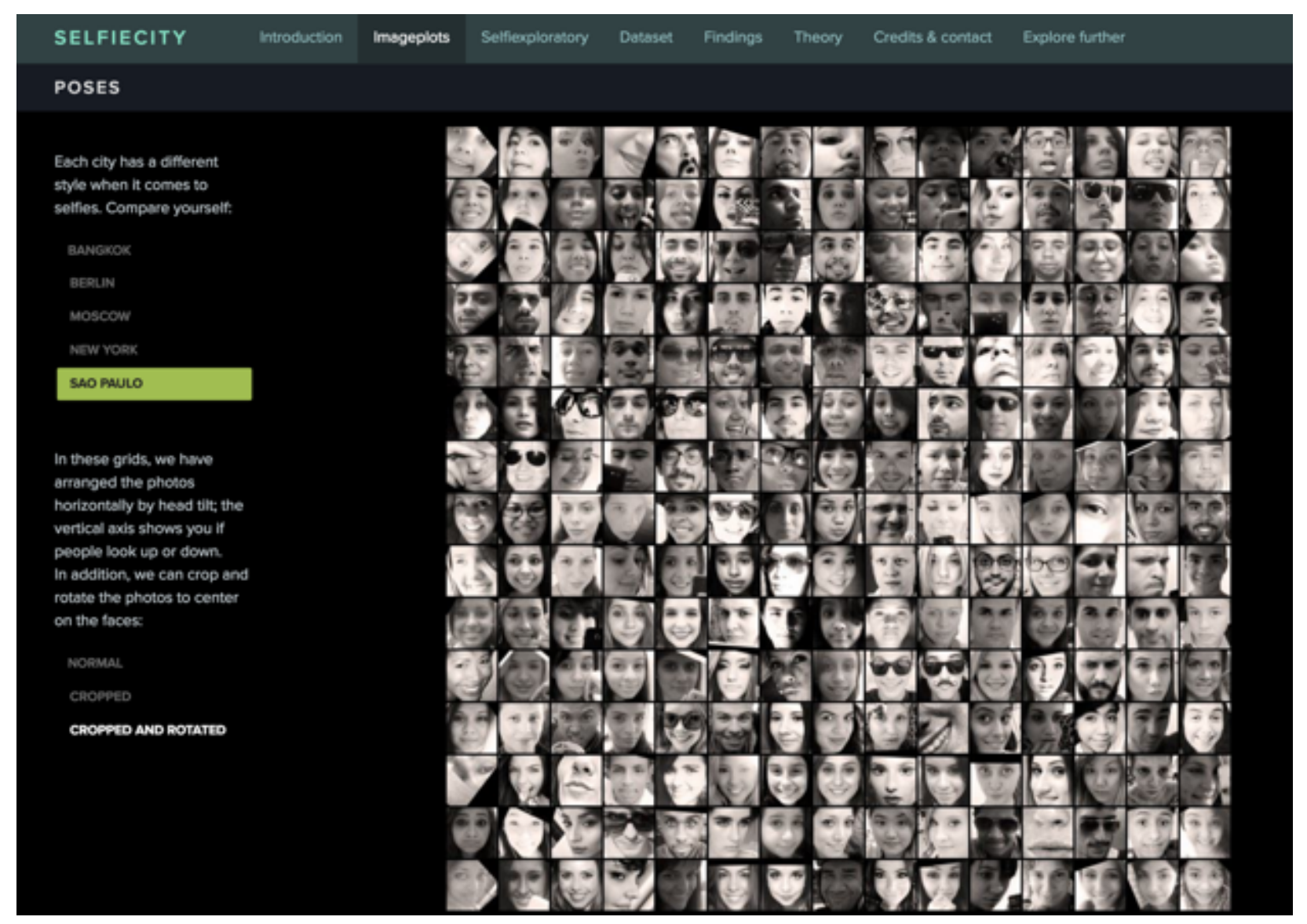

Figure 5: Selfiecity (Manovich et al., 2014)

Through an analysis of images and associated text, we identify elements of the selfie gaze and how this practice contributes to the production and evolution of the digital cultures of everyday life. The examples we show here feature selfies of ourselves which are representative of our findings but are not the images we actually reviewed. While the selfies we used are publically available, the nature of the images - focused on the self and private actions aimed primarily at the limited confines of a person's social network - led us to exercise an abundance of caution in reproducing them here. We also did this to highlight the emerging practices and debates around responsible big data research as something that should be a part of any similar research (Zook et al, 2016).

Although there are interesting and nuanced differences between selfie practices in Indonesia and the United States, it immediately stands out how different many selfies are from those represented in the Selfiecity. Most striking perhaps, is that many selfies (see Figure 6b) are not only about the self (see Figure 6a). Other people included within the selfie ('we-fie') are in many cases $(60 \%)$ people that are significant to the author of the selfie. With the selfie, not 
only is the self represented but also relationships to other significant people to the self. This can range from a night out on the town with your best friend, to a baseball game with your father or the birthday of your great-grandmother. In addition to people, selfies also provided spatial context and connection to places, only 18 percent were lacking this. Although for the majority of coded images ( 60 percent in total) the connection to place was relative banal - the train station; the bar; at school, etc. - this represents an important documentation of everyday life. The final 20 percent of selfies were coded as representing a connection to a significant place, for example a famous tourist destination or landmark (Figure 6c).
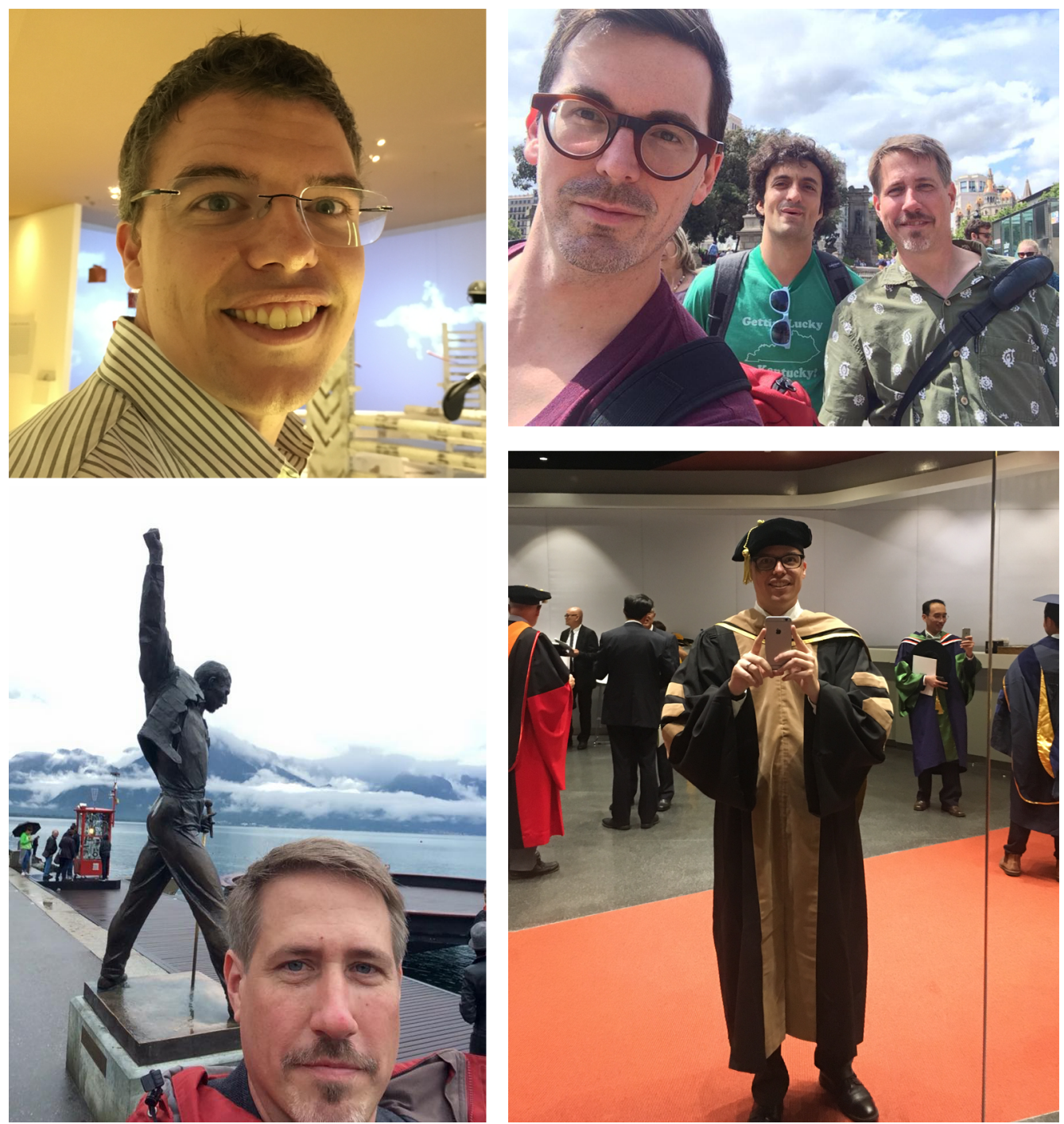

Figure 6a-d Different 'types' of selfies

Some contrary to our expectations the comparison of selfies from Indonesia and the United States shows relatively congruous practices. While differences in dress are apparent - most notably the wearing of a hijab by women in Indonesia, which appeared in 30 percent of these 
selfies - there are ample examples in both countries of people taking either solitary or group pictures that show banal and significant places and events in their lives. In other words, the general impression that emerged from our coding was that the multi-faceted practice of selfies in both countries is remarkably similar, including the frequent flashing of the $\mathrm{V}$-sign. In other words, selfies were used as part of complex and cultural-referenced conversations in which the relationships and spatial situatedness of the self played key roles. Although we highlight some differences between the practices in both countries in this section, ultimately these practices as conversations are much more similar to each other than to the stereotypical selfie - self-indulgent vanity - described in popular media.

It is important to note that other variables in our coding process reinforced the difficulty of interpreting digital cultural texts (Rose, 2016) particularly when a language barrier was present. For example, categorizing an event pictured in a selfie as either banal or significant was more easily performed when we were able to understand the accompanying text. Simple strings like "birthday" or "reunion" or "graduation" quickly enable us to code a selfie as a significant event while similar strings in Indonesian were indecipherable. That said, even with English text, we are certain that our interpretation of some selfies is inconsistent with the intended message of the sender given the other dimensions of cultural difference.

Despite these short-comings some differences in selfie practice is evident between the two countries as outlined in Table 3 (focused on the demographics of the individuals featured in the photos) and Table 4 (focused on practices). Selfies are significantly more a solitary practice in the United States, as over 72 percent of images in our sample were of a single individual. In contrast, selfies from Indonesia were more than twice as likely to feature more than two people and only half of all selfies from Indonesia only have a single person visible. Likewise, we observed differences in the age of people featured in selfies but are cautious in this assessment as determining age based only on visible facial features is problematic. Nevertheless, the magnitude of this difference - 90 percent of Indonesian selfies were coded as featuring only individuals less than 30 while the same figure was 65 percent for the US gives confidence that this is a real variance. A final category of difference was sex - also a difficult categorization based on our visual assessment - but we observe variation in that selfies featuring just males were significantly more prevalent in the US than Indonesia. 


\begin{tabular}{lrrrr}
\multicolumn{5}{c}{ Differences in Demographics } \\
Number of People in Selfie \\
Category & Indonesia & USA & Z-score & Sig. \\
1 & $51.6 \%$ & $72.1 \%$ & -4.36 & $* *$ \\
2 & $25.4 \%$ & $18.1 \%$ & 1.81 & \\
Group & $23.0 \%$ & $9.8 \%$ & 3.7 & $* *$
\end{tabular}

$\begin{array}{lcccc} & \text { AGE } & & & \\ \text { Category } & \text { Indonesia } & \text { USA } & \text { Z-score } & \text { Sig. } \\ <30 & 90.6 \% & 64.7 \% & 6.44 & * * \\ >30 & 1.9 \% & 19.1 \% & -5.8 & * * \\ \text { Multiple/Unknown } & 7.5 \% & 16.3 \% & -2.8 & * *\end{array}$

\begin{tabular}{lcccc} 
& \multicolumn{2}{c}{ SEX } & & \\
Category & Indonesia & USA & Z-score & Sig. \\
All Female & $56.3 \%$ & $54.0 \%$ & 0.5 & \\
All Male & $16.0 \%$ & $23.7 \%$ & -2.01 & $*$ \\
Mixed & $23.0 \%$ & $17.2 \%$ & 1.5 & \\
Unknown & $4.7 \%$ & $5.1 \%$ & -0.2 &
\end{tabular}

Table 3. Differences in Selfie Demographics between Indonesia and the USA

While we do not wish to read too much into these differences, they are consistent with broad understandings of the ways in which these countries' digital cultures have developed. Indonesia has a much younger population than the US and has more recently joined the "information society/economy". Thus, the younger demographic represented in selfie culture may simply be an artifact of the country's development history, although this is far from certain. Likewise, the practice of more solitary selfies in the US might lead us to speculate that this is a reflection of a more individualistic society in which selfies act as efficient oneto-one messages or one-to-many broadcasts in contrast to the more communal culture within Indonesia. But these are preliminary and speculative hypotheses rather than conclusions. 
Table 4 shows that more significant events are represented in US versus Indonesian selfies. We report this, however, primarily to demostrate problems inherent in our coding rather than to present it as an established fact. When this difference first emerged from our analysis, we went back to the already coded selfies and realized that our ability to judge whether a selfie shows a significant event is greatly assisted by the text within the tweet. Since we are unable to read a selfie tweet from Indonesia in a similar manner (neither of us speak Bahasa Indonesia), it is extremely likely that Indonesia is under-coded in this respect. Despite this difficulty in reading cultural texts across languages we were able to identify other clear differences in cultural practices between the countries.

The first of such practices is the use of the collage effect - in which several selfies are tied together or in which "stickers" are applied to a photo - was significantly more prevalent in Indonesia than the US (Figure 7a). These collages are often enabled by a myriad of thirdparty selfie applications and services, which allow the user to apply filters or in which new stickers can be purchased in 'packs'. While we are uncertain of the cause - is it tied to software, a marketing campaign, etc. - it clearly demonstrates how the conversations mediated through selfies can be augmented and deepened if the prevailing digital culture develops the norm of doing so. This ability is perhaps best illustrated by Snapchat's - the ephemeral messaging platform - face 'lenses'. The application embeds powerful face recognition software, which then is used to add on masks to the users face, swap faces with a friend or augment the photo or video message in other ways.

In a similar manner, the US has its own practice, which we name solicitation, that was not found at all in Indonesia. This practice evident in ten percent of US selfies in our sample consists of someone - often with celebrity status or at least with a large number of followers - sending out a tweet that says "[target audience] quote(or retweet) this with your [adjective] selfie". The goals of these message are multi-faceted and complex - including marketing, gathering prurient or funny images, etc. - but the commonality is an effort to gain attention for both the initial sender and the retweeters. Example include, "ladies quote this with your sexy selfie" or "fans retweet this with a selfie showing your fandom" (Figure 7b). 


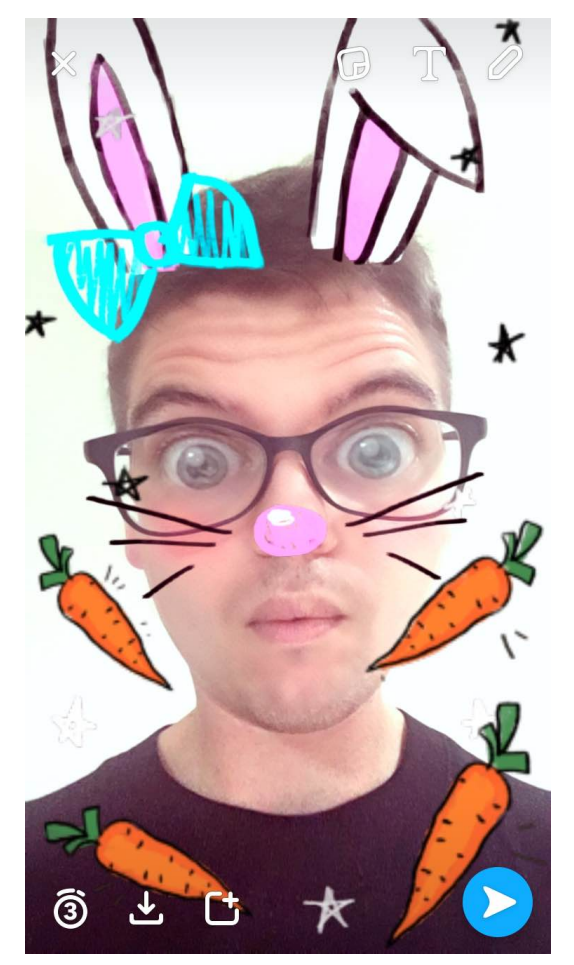

Stay Positive @cupcakeposi·sep 15

If you reply to this with a selfie, I will quote it with what I find beautiful about you! \#Love \#Positivity

Figure 7 Examples of collage effect in selfies (a) and solicitation of selfies (b)

\begin{tabular}{lcccc}
\hline \multicolumn{5}{c}{ Differences in Practices } \\
Category & Indonesia & USA & Z-score & Sig. \\
none & $71.8 \%$ & $48.8 \%$ & 4.86 & $* *$ \\
banal & $16.9 \%$ & $27.0 \%$ & -2.52 & $*$ \\
significant & $11.3 \%$ & $24.2 \%$ & -3.5 & $* *$ \\
& & & & \\
& & & & \\
Category & Indonesia & USA & Z-score & Sig. \\
No Collage & $71.4 \%$ & $93.0 \%$ & -5.86 & $* *$ \\
Collage & $28.6 \%$ & $7.0 \%$ & 5.86 & $* *$ \\
& & & \\
& & & \\
\hline
\end{tabular}




$\begin{array}{lcccc}\text { Category } & \text { Indonesia } & \text { USA } & \text { Z-score } & \text { Sig. } \\ \text { No Solicitation } & 100.0 \% & 89.8 \% & 4.79 & * * \\ \text { Solicitation } & 0.0 \% & 10.2 \% & -4.79 & * *\end{array}$

Table 4. Differences in Selfie Practices between Indonesia and the USA

The difference in practice exemplified by collaging and soliciting shows how the same technology can be used in widely different ways depending upon the cultural context in which it is embedded.

We must also acknowledge that differences between the two countries are not the only ones that matter. For example, when we consider the differences in selfies featuring men or women (regardless of country) we note that a higher percentage of selfies featuring men (29.4\% vs $16.5 \%$ ) have spatial context or connections to place that we coded to be significant (versus banal or indistinct). While recognizing the potential for error in this coding, this difference suggests a gendered dimension of selfie practice in which women are more likely to use selfies firmly within the context of their everyday lives' and thus featuring what we coded as connections to the quotidian. To be sure, this category itself is problematic as what might seem as banal to outsiders (such as ourselves) might actually have deep significance, e.g. the commonplace interior wall we see could be one's best friend's or grandmother's house.

\begin{tabular}{lcccc}
\hline \multicolumn{5}{c}{ Differences Between Gender Practice and Places } \\
Significant place in Selfie \\
Category & Female & Male & Z-score & Sig. \\
none & $20.3 \%$ & $12.9 \%$ & 1.51 & \\
banal & $63.1 \%$ & $57.6 \%$ & 0.89 & \\
significant & $16.5 \%$ & $29.4 \%$ & -2.55 & $*$
\end{tabular}

Table 5. Differences in Featuring Significant Places in Selfies featuring Females or Males 
In this analysis we are less concerned about the exact typologies and definitions we create, and more focused on showing some of the nuances and differences in the production and interpretation of digital culture and illustrating how these are dependent upon one's own gaze or subjectivity. It is certainly possible to be more systematic in creating a categorization and coding. In fact, many of the quantitative explorations of selfies that we touched on earlier are examples of such systematic approaches. Arguably, these approaches can miss substantial aspects of selfie culture exactly because of their a-priori categorization and our work foregrounds the uncertainties and subjectivities of such classifications. Sometimes this uncertainty can be fairly well understood, i.e., unknown languages confound interpretation, while other times this remain much less certain, gauging the meaningfulness of a photo backdrop to its sender. But conversations - digital, visual or otherwise - are key parts of human experience and culture and thus are key topics for research.

\section{Conclusion}

In this chapter, we have explored the geography of the selfie from various perspectives. As our examples have shown, it is abundantly clear that selfies are indeed not just a symptom of the narcissistic 'me' culture of today's millennial generation. Selfies form, for many people, an integral aspect of daily life. They are not just used as a means to represent or present the self to the outside world. Selfies are a means of conversing with one another and with the world. That conversation, of course, includes the self, but it also includes other people, places (banal and special alike) and events. In this way, selfies are like many other technologymediated phenomena: they are part and parcel of the production and consumption of social space.

Our quantitative analysis and 'mapping' of selfies in geographic space shows that the intensity with which selfies are used differs greatly around the world. Perhaps surprisingly, selfies are a global phenomenon, relatively popular outside of the Anglophone world with Bangladesh, Cambodia and the Philippines as the top-3 selfie countries on Twitter (normalized by total number of tweets sent from each country). While the mapping of selfies shows the pervasiveness of the practice around the world, the qualitative analysis of the actual selfies from our case studies of Indonesia and the United States shows both significant differences in the types of selfies sent as well as the connections that selfies build with local places. 
On a methodological note, the two approaches used in this chapter show the usefulness of combining qualitative and quantitative methods and the complementary fit between the projectionist and perspectivalist view on the world. This two perspectives are not mutually exclusive. Much like how $17^{\text {th }}$ century atlases included both projectionist maps and perspectivalist drawings of lands wide-and-far, taken together these two perspectives on selfie culture paint a much more complete and illustrative picture than a single perspective would be able to reflect by itself.

\section{Bibliography}

Bakhshi, S., Shamma, D. A., Gilbert, E., Bakhshi, S., Shamma, D. A., \& Gilbert, E. (2014). Faces engage us: photos with faces attract more likes and comments on Instagram. the 32nd annual ACM conference (pp. 965-974). New York, New York, USA: ACM. http://doi.org/10.1145/2556288.2557403

Boy, J. D., \& Uitermark, J. (2015, August). Capture and share the city: Mapping Instagram's uneven geography in Amsterdam. In RC21 International Conference on "The Ideal City: Between Myth and Reality. Representations, policies, contradictions and challenges for tomorrow's urban life," Urbino, Italy.

Boy, J. D., \& Uitermark, J. (2016). How to Study the City on Instagram. PloS one, 11(6), e0158161.

Busetta, L., \& Coladonato, V. (2015). Introduction Be Your Selfie: Identity, Aesthetics and Power in Digital Self-Representation. Networking Knowledge: Journal of the MeCCSA Postgraduate Network, 8(6).

Dinhopl, A., \& Gretzel, U. (2016). Selfie-taking as touristic looking. Annals of Tourism Research, 57, 126-139. http://doi.org/10.1016/j.annals.2015.12.015

Ellison, N. B., Vitak, J., Steinfield, C., Gray, R., \& Lampe, C. (2011). Negotiating privacy concerns and social capital needs in a social media environment. In Privacy online (pp. 19-32). Springer Berlin Heidelberg.

Farci, M., \& Orefice, M. (2015). Hybrid Content Analysis of the Most Popular Politicians' Selfies on Twitter. Networking Knowledge: Journal of the MeCCSA Postgraduate Network, 8(6). 
Franco, J. (2013). The meanings of the selfie. The New York Times, 28.

Hjorth, L., \& Hendry, N. (2015). A snapshot of social media: Camera phone practices. Social Media+ Society, 1(1), 2056305115580478.

Hochman, N., \& Manovich, L. (2013). Zooming into an Instagram city: Reading the local through social media. First Monday. http://firstmonday.org/ojs/index.php/fm/article/view/4711

Lyu, S. O. (2016). Travel selfies on social media as objectified self-presentation. Tourism Management, 54, 185-195.

Manovich, L., M. Stefaner, M. Yazdani, D. Bauer, D. Goddemeyer, A. Tifentale, N. Hochman, and J. Chow (2014). selfiecity. http://www.selfiecity.net/. Last accessed on October 1 2016

Marwick, A. E. (2015). Instafame: Luxury selfies in the attention economy. Public Culture, 27(1 75), 137-160.

OxfordDictionary. (2013). The Oxford Dictionaries Word of the Year 2013. November 19. http://blog.oxforddictionaries.com/press-releases/oxford-dictionaries-word-of-the-year2013/

Poorthuis, A., Zook, M., Shelton, T., Graham, M., and Stephens, M. (2016) Using Geotagged Digital Social Data in Geographic Research, in: Clifford, N., French, S., Cope, M., and Gillespie, T. (eds) Key Methods in Geography, 3rd Ed., London: Sage.

Poorthuis, P. and M. Zook. 2016. Making Big Data Small: Strategies to Expand Urban and Geographical Research Using Social Media. (Under review at the Journal of Urban Technology).

Rose, G. (2012). Visual Methodologies. London: Sage.

Rose, G. (2015). Rethinking the geographies of cultural 'objects' through digital technologies: Interface, network and friction. Progress in Human Geography. doi:10.1177/0309132515580493

Rose, G. (2016). Cultural geography going viral. Social \& Cultural Geography, 1-5.

Saltz, J. (2014). Art at arm's length: A history of the selfie. New York Magazine.

Shelton, T., A. Poorthuis. Zook, M. (2015). Social media and the city: Rethinking urban sociospatial inequality using user-generated geographic information. Landscape and Urban Planning. pp. 198-211 doi:10.1016/j.landurbplan.2015.02.020 
Souza, F., Las Casas, de, D., Flores, V., Youn, S., Cha, M., Quercia, D., \& Almeida, V. (2015).

Dawn of the Selfie Era: The Whos, Wheres, and Hows of Selfies on Instagram. the 2015 ACM (pp. 221-231). New York, New York, USA: ACM.

http://doi.org/10.1145/2817946.2817948

Tifentale, A., \& Manovich, L. (2015). Selfiecity: Exploring photography and self-fashioning in social media. In Postdigital Aesthetics (pp. 109-122). Palgrave Macmillan UK.

Urry, J. (2002). The tourist gaze. London: Sage.

Wargo, J. M. (2015). "Every selfie tells a story...": LGBTQ youth lifestreams and new media narratives as connective identity texts. New Media \& Society, 1461444815612447.

Zook, M. and A. Poorthuis. (2014). Offline Brews and Online Views: Exploring the Geography of Beer Tweets. Chapter in M. Patterson and N.Hoalst-Pullen Geographies of Beer. Springer. 201-209

Zook, M. (+10 other authors, final list is pending). 2016. Ten Simple Rules for Responsible Big Data Research. (under review at PLOS ONE). 